\title{
Plasma density from Cerenkov radiation, betatron oscillations, and beam steering in a plasma wakefield experiment at $\mathbf{3 0}$ $\mathrm{GeV}$
}

\author{
P. Catravas, S. Chattopadhyay, E. Esarey, W.P. Leemans
}

Lawrence Berkeley National Laboratory, Berkeley, CA 94720

R. Assmann*, F.-J. Decker, M.J. Hogan, R. Iverson, R.H. Siemann, D. Walz, D. Whittum

Stanford Linear Accelerator Center, Stanford University, Stanford CA 94309

*present address: CERN, Geneva, Switzerland

B. Blue, C. Clayton, C. Joshi, K. Marsh, W.B. Mori, S. Wang

University of California Los Angeles, Los Angeles CA 90095

T. Katsouleas, S. Lee, P. Muggli

University of Southern California, Los Angeles CA 90089

\begin{abstract}
Measurements of radiation near the $670.8 \mathrm{~nm}$ atomic spectral line of Lithium as a $30 \mathrm{GeV}$ electron beam propagates through a long, high density heat pipe oven $\left(\sim 1\right.$ meter, neutral density $\left.\sim 10^{15} \mathrm{~cm}^{-3}\right)$ have been performed. Practical considerations in the use of Cerenkov radiation as an automatically synchronized diagnostic of the plasma source properties are investigated. Parametric scans of the Cerenkov spatial profile (temperature, pressure, wavelength and laser/ebeam delay) are presented and the source neutral and plasma density are estimated from the measurements. Large increases in the number of intercepted photons are noted in the presence of plasma near strong spectral lines which depend on laser/e beam timing and steering.
\end{abstract}




\section{INTRODUCTION}

Experimental efforts in plasma wakefield interactions strive for increased accelerating gradients and interaction lengths as well as improved control over the output electron beam $[1,2]$. The plasma properties, both integrated and time resolved along the electron bunch, are a determining factor in vital aspects of the interaction. The optimum wakefield is achieved when the length of the driving pulse (electron bunchlength or laser pulselength) is on the order of the plasma wavelength. Accurate measurement of plasma density is essential to interpreting experimental studies of the transformer ratio, transverse focussing strength and the growth rate of instabilities. Standard plasma diagnostics often rely on an external laser to sample the properites of the plasma source using interferometric or absorption methods. However, radiation produced by the interaction of the electron beam with a medium provides special opportunities for diagnosis of source properties. Such a radiation pulse co-propagates with the electron beam, sampling the source exactly along the path travelled by the beam with perfect temporal synchronization, down to the timescale of the beam bunchlength. Because this radiation is broadband, the observation wavelength can be selected as needed for flexible diagnostic design. Here we describe experiments in plasma source characterization using Cerenkov radiation in which we design the diagnostic through choice of the observation wavelength with respect to an atomic spectral line.

We will describe measurements of the spatial profile of Cerenkov radiation for the characterization of the plasma properties for the E157 Plasma Wakefield Acceleration Experiment at the Stanford Linear Accelerator Center. A 2 ps (rms) long electron beam bunch with $2 \times 10^{10}$ particles in a $30-100 \mu \mathrm{m}$ spot propagates through a Lithium plasma 1.4 meters long. The electron bunch blows out plasma electrons, resulting in transverse focussing forces along the bunch, and longitudinal accelerating forces as the plasma electrons return at the electron bunch tail. The nominal plasma density of $2 \times 10^{14} \mathrm{~cm}^{-3}$ is set according to a tradeoff between the maximum longitudinal field and the fraction of the tail electrons which can experience acceleration. Measurements of Cerenkov radiation near the $670.8 \mathrm{~nm}$ spectral line of Lithium are presented in which the radiation is studied as a function of temperature, observation wavelength and relative timing of the laser and electron beam. Neutral density and plasma density are extracted from these measurements $[3]$.

\section{DIAGNOSTIC SCHEME}

Cerenkov radiation [4] is produced when the speed of light in the medium, $c / n$, drops below that of the electrons, $u$. The index of refraction of the medium contains a strong positive contribution from atomic spectral lines on the long wavelength 
side of the resonance, which can exceed the negative contribution from plasma electrons and ions, and set the index of refraction well above $\beta=u / c$ for a broad region of the spectrum. Alternately, Cerenkov radiation will disappear for specific portions of the spectrum, particularly near the short wavelength side of a spectral resonance, where the index of refraction drops below $\beta$. The phase accumulation of the co-propagating radiation is proportional to the change in index of refraction, $n-1$, of the medium through which it propagates.

The diagnostic scheme is shown in Figure 1. The electron beam propagates through a medium of length $2 X$, and a thin foil outcouples the radiation downstream. The spectral flux density of Cerenkov radiation, written below in the far field for $n-1$ positive [4],

$$
\frac{d U_{\omega}}{d \Omega}=\frac{e^{2} n \omega^{2} \beta^{2}}{4 \pi^{3} \epsilon_{0} c} \sin ^{2} \theta \frac{\sin ^{2}(1-n \beta \cos \theta) \frac{\omega X}{\beta c}}{\frac{\omega^{2}}{(\beta c)^{2}}(1-n \beta \cos \theta)}
$$

is sensitive to changes in the medium index of refraction. Here, e, $\omega$, and $\epsilon_{0}$ are the electron charge, observation frequency, and permettivity of free space. For $\sin ^{2} \theta$ slow varying compared to the sinc function, i.e. $\sin ^{2} \theta \sim\left(2 / n^{2}\right)\left(n-1+\frac{1}{2 \gamma^{2}}\right)$, phase matching occurs at the angle $\theta_{c}$ given by

$$
\frac{\theta_{c}^{2}}{2} \sim n-1-\frac{1}{2 \gamma^{2}} .
$$

Neglecting absorption, the medium index of refraction can be modelled by,

$$
n-1 \sim \sum_{\text {species }, s} \frac{1}{2} \frac{N_{s} e^{2}}{m_{e} \epsilon_{0}} \frac{f_{i k}}{\omega_{r e s}^{2}-\omega^{2}}-\frac{\omega_{p e}^{2}}{2 \omega^{2}}
$$

where $N_{s}$ is the species density, $e$ and $m_{e}$ are the electron charge and mass, $f_{i k}$ is the oscillator strength, $\omega_{\text {res }}$ is the frequency of the atomic resonance, $\omega$ is the observation frequency, and $\omega_{p e}$ is the electron plasma frequency.

Choosing the observation wavelength appropriately with respect to an atomic spectral line allows measurement of the source parameters. By invoking various limits of Eqns. 2 and 3, the contributions from neutral density, plasma density, and various beam parameters may be separated and diagnosed. The shift in spectral lines upon ionization allows any single species, ions or neutrals, to be selected via observation wavelength on the red side of its spectral resonance. When $1 / \gamma^{2}<<$ $n-1$ and the plasma electron density, $N_{e}$, is much less than the critical density, $N_{\text {crit }}\left(\omega_{p e}{ }^{2}=N_{\text {crit }} e / m_{e} \epsilon_{0}\right)$, the square of the cone angle is directly proportional to the neutral or ion density. A second method for plasma density measurement is through monitoring the fractional ionization of the neutrals: $\left(N_{i} / N_{n}\right)$, where $N_{i}$ is the ion density. A baseline measurement without plasma provides the initial neutral density. The amount of cone angle reduction quantifies the depletion of the neutral population, $N_{n}-N_{i}$. The ratio of the two cone radii will be proportional to 


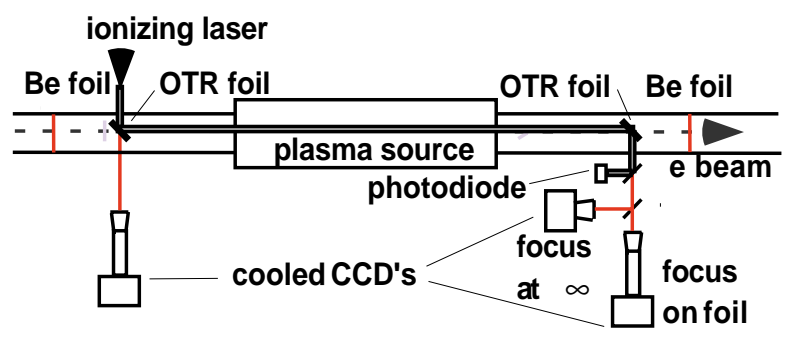

FIGURE 1. Experimental setup

$\sqrt{1-N_{i} / N_{n}}$. Two shots therefore provide both neutral and plasma density. Note that through choice of frequency, $\omega$, such that $N_{s} e^{2} f_{i k} /\left(m_{e} \epsilon_{0}\left(\omega_{r e s}^{2}-\omega^{2}\right)\right) \sim \omega_{p e}^{2} / \omega^{2}$, the electron plasma density can be measured directly using the Cerenkov radiation threshold. The implementation of the method of neutral depletion is presented below.

\section{EXPERIMENTAL RESULTS}

In the E157 plasma wakefield acceleration experiment [5] at the Stanford Linear Accelerator FFTB, a $30 \mathrm{GeV}$ electron beam with $1-2 \times 10^{10}$ particles propagates through a heat pipe oven [6], [7] which provides a Lithium plasma of density $\sim 10^{14}$ $\mathrm{cm}^{-3}$ (Fig. 1). The typical Lithium column length is 1.4 meters plugged by Helium at a partial pressure of about 0.45 Torr. The Lithium neutral density is above $10^{15}$ $\mathrm{cm}^{-3}$. The ionizing laser, an ArF Excimer operating at $193 \mathrm{~nm}$, is incoupled and outcoupled by fused Silica mirrors, $150 \mu \mathrm{m}$ thick, separated by 2.5 meters. One side is coated with Aluminum for electron beam spot size monitoring using OTR [8]. The opposite side contains a high reflecting dielectric coating at $193 \mathrm{~nm}$. Images with focus at the foil or focus at infinity of the radiation upstream and downstream are measured with 14 and 16 bit cooled CCD's. The plasma density decays on a timescale of about $10 \mu \mathrm{sec}$. The plasma density can therefore by changed from shot-to-shot by varying the time between laser and electron beam injection.

The main spectral lines of interest for this measurement are those of the ground states of neutral Lithium $(670.8 \mathrm{~nm})$, singly ionized Lithium $(19.9 \mathrm{~nm})$, and neutral Helium $(58 \mathrm{~nm})$. In spite of the presence of multiple species, it is not difficult to separate out the cone of neutral Lithium, as the strong dependence of the cone radius on wavelength near $670.8 \mathrm{~nm}$ can be used to set the cone at a few mrad, and avoid any potential overlaps. When $n-1>>1 /\left(2 \gamma^{2}\right)$ a change in index of refraction of only one part in $10^{6}$ results in $\theta_{c}=1 \mathrm{mrad}$. Wavelengths are sufficiently close to the spectral line that the contribution from plasma electrons be negligible. For a $30 \mathrm{GeV}$ electron beam, the contribution from $1 / 2 \gamma^{2}, 1$ part in $10^{9}$, is much smaller than $n-1$ across the entire visible spectrum.

Scans with the imaging focal plane located at the foil $0.65 \mathrm{~m}$ downstream from end of oven and far field (focus at infinity) were performed during oven cool down 


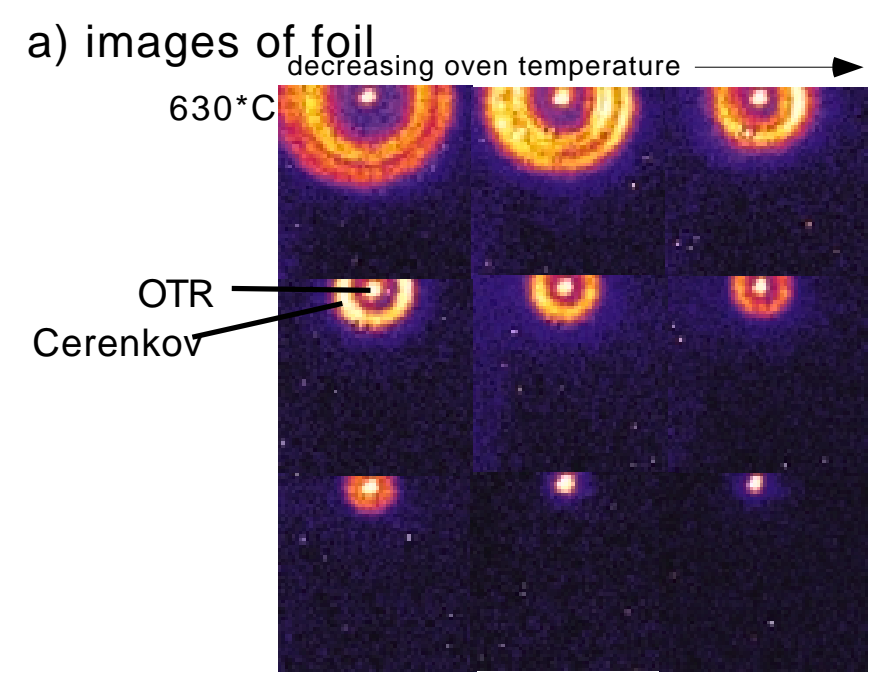

b) far field decreasing oven temperature

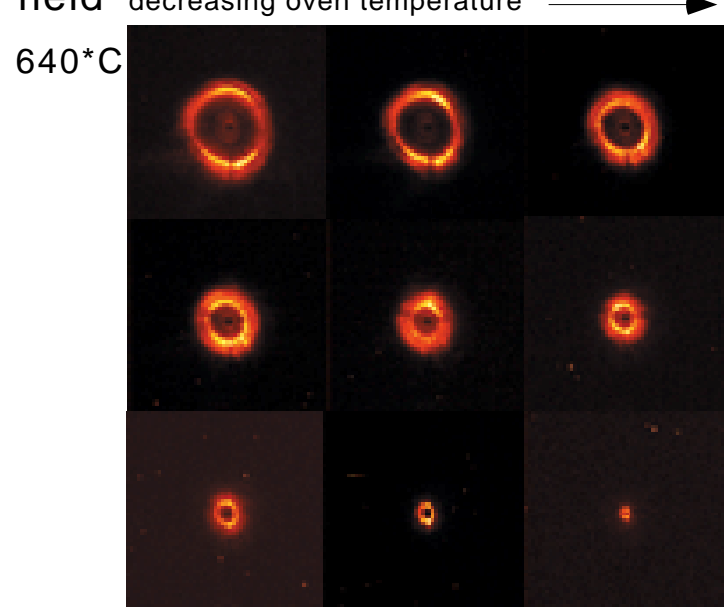

$\lambda=676.4 \mathrm{~nm}, \mathrm{Li}: \lambda_{\text {res }}=676 \mathrm{~nm}$,

filter: $700 \mathrm{~nm}, \mathbf{4 0} \mathrm{nm}$ BW

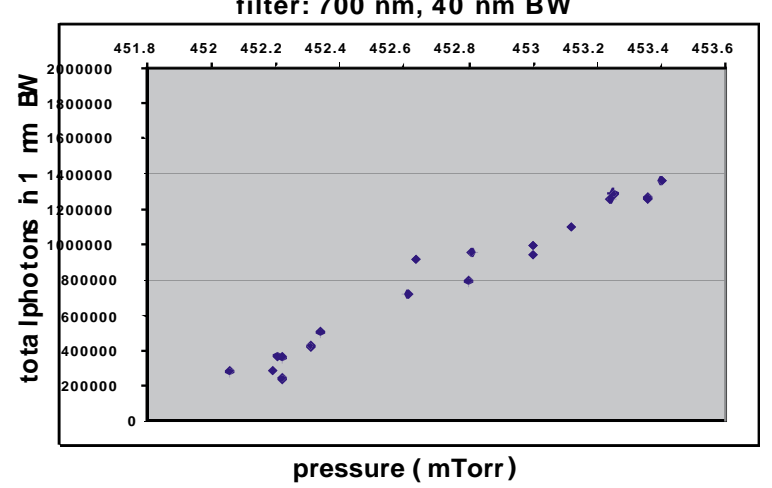

FIGURE 2. Images of the foil downstream of the oven (a) and corresponding far field images (b) provide an estimate of the Lithium neutral density as the oven temperature is changed. Observation wavelength $676 \mathrm{~nm}$; max oven temperature $630^{\circ} \mathrm{C}$. c) Intercepted photon level at $700 \mathrm{~nm}$ as a function of Helium buffer pressure and oven exterior shield temperature during an oven temperature scan is in reasonable agreement with theory. 


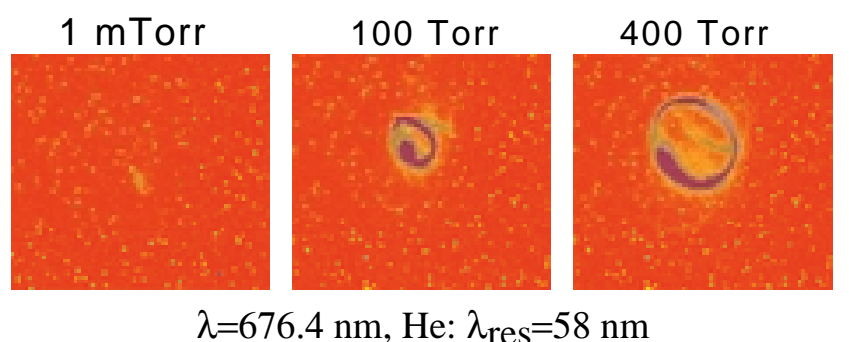

FIGURE 3. Far field images with a single gas, Helium, filling the oven at room temperature. Even $600 \mathrm{~nm}$ away from the spectral line, the cone radius responds to changes in density. An independent measure of the density from a pressure gauge agreed with the density extracted from the Cerenkov cone.

a)
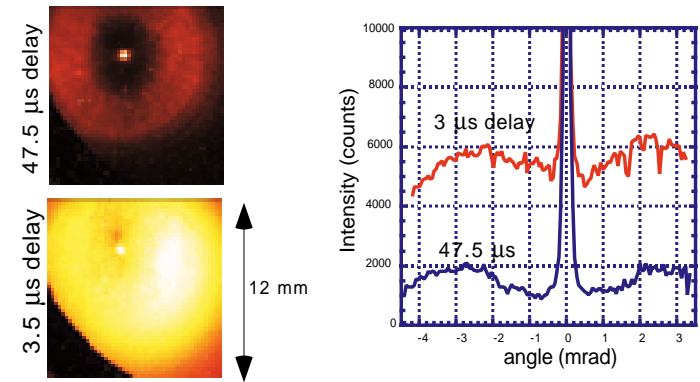

b)

c)
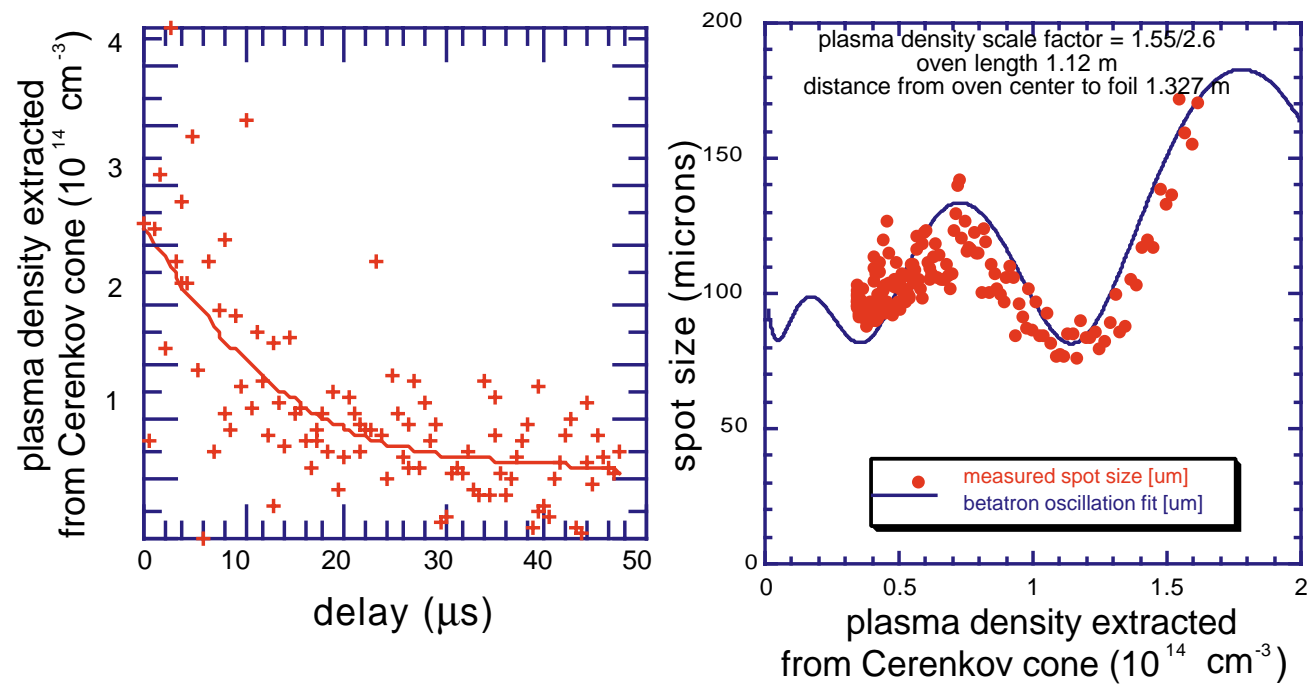

FIGURE 4. Images of the downstream spatial profile are compared for two laser/electron beam delays: 3 and $47.5 \mu s$ (a). The Cerenkov cone shifts inward atop a large visible background when the plasma density is large. From measurements of the Cerenkov cone radius as a function of delay, the evolution of the plasma density was estimated (b). Plasma density estimated from Cerenkov radiation is in reasonable agreement with measured betatron oscillations (c). 
to monitor the dependence of the Cerenkov spatial profile on neutral density. The results for an observation wavelength of $676+/-0.75 \mathrm{~nm}$, shown in Fig. 2a and $\mathrm{b}$, show the systematic response of the cone peak angle to the Lithium neutral density. The central spot in the images in Fig. 2a is mainly OTR (i.e. an image of the transverse profile of the electron beam), and remains unchanged in the images even when the Lithium vapor pressure is very low and the Cerenkov cone is no longer detectable. The neutral density scaling with temperature, $\mathrm{T}$, as the Lithium vapor changes state is

$$
N_{n} \sim T^{-1} \exp (-\phi / k T)
$$

where $\mathrm{k}$ is the Boltzmann constant. The logarithm of these measured cone peak angles vary linearly with inverse temperature with negative slope, as expected. The peak neutral density extracted for this scan was $2.1 \times 10^{15} \mathrm{~cm}^{-3}$ at for an exterior shield temperature of $630^{\circ} \mathrm{C}$. The Helium buffer pressure was 270 mTorr. The photon count measured during an oven temperature scan for a $40 \mathrm{~nm}$ bandwidth centered at $700 \mathrm{~nm}$ is shown in Fig. 2c. The measurement lies within a factor of two of the theoretical prediction [4], which is within the error bar due to attenuation factors and background subtraction.

As a benchmark, measurements of Cerenkov radiation with the oven filled only with Helium at room temperature (Fig. 3) were performed at wavelengths $600 \mathrm{~nm}$ longer than the main spectral line. The Helium density was estimated using known values of the index of refraction [9] in the visible, and compared to the density given by a pressure gauge at 1 mTorr, 100 Torr and 400 Torr. The two measurements agreed to within $10 \%$. The error is probably due to residual cylindrical focussing from a splitting pellicle in the system, which affected the cone aspect ratio by $10 \%$. Systematic studies of a long Helium column have been performed by other groups [10] in the FFTB beamline at SLAC.

The Lithium plasma density was varied by changing the relative delay between the electron time of arrival and the time of fire of the ionizing laser. Fig. 4a compares images of the downstream foil for an electron beam time of arrival $48 \mu$ s after laser fire and immediately after laser fire. Two effects are seen: an inward shift of the Cerenkov cone and a substantial increase in background radiation. The cone radius was measured for a laser timing scan from extending from the time of laser fire to $50 \mu \mathrm{s}$. The observation bandwidth was $675 \mathrm{~nm}+/-0.75 \mathrm{~nm}$. The separation of the Cerenkov cone from the visible background was performed by incremental subtraction of images taken at adjacent time steps. The plasma density extracted from the inward shift of the cone peak angle as a function of time after laser fire is shown in Figure 4b. Close to zero delay, the estimated plasma density was about $2.6 x 10^{14} \mathrm{~cm}^{-3}$. This number is in reasonable agreement with plasma densities estimated from simultaneous measurements of betatron oscillations downstream from the oven and UV absorption [3]. The use of betatron oscillations as a plasma density diagnostic has been carefully studied [11]. The measured betatron oscillations which accompanied the data of Fig. $4 \mathrm{~b}$ are shown in Fig 4c. Given oven lengths 
from 1.4 to 1.0 meters, the fits yielded maximum initial plasma densities from 1$2 \times 10^{14} \mathrm{~cm}^{-3}$. The background was found to vary not only with laser/electron beam delay but also laser steering. As a result, it cannot be explained by recombination radiation from the laser ionized plasma over the 0.5 second camera integration time. We speculate that the visible background is due to increased recombination radiation resulting from wakefield energy dissipation through excitation and ionization of the component species, leaking through the tail of the spectral response of the interference filter at $670.8 \mathrm{~nm}[12]$.

In general, the choice of wavelength is set by tradeoffs in diagnostic accuracy. When the wavelength is set too close to the spectral resonance shifts of the resonant frequency (Stark/Zeeman) due to high electric and magnetic field need to be included and uncertainty increases because of dispersion. Far away from resonance, care must be taken to model the index of refraction correctly, and eventually the Cerenkov threshold condition occurs. However, the working range for the spatial profile can be large; for our plasma parameters, the threshold condition does not occur until the infrared wavelengths. By providing sufficient extinction for backgrounds higher than initial plasma recombination as described above, and making use of the full CCD frame (260,000 pixels), substantial improvements in the accuracy of the plasma density measurement can be easily achieved.

\section{CONCLUSION}

A method for using Cerenkov radiation near atomic spectral lines to measure plasma source properties for plasma wakefield applications has been discussed and experimentally verified. Because the radiation co-propagates with the electron beam, the radiation samples the source properties exactly along the path of interest with perfect temporal synchronization. Observation wavelengths were chosen with respect to the atomic resonances of the plasma source, where the relative change in the index of refraction strongly affects the Cerenkov cone angle, and permits flexible diagnostic design. The Cerenkov spatial profiles were systematically studied for a Lithium heat pipe oven as a function of oven temperature and observation wavelength. Neutral densities and plasma densities were extracted from the measurements.

\section{ACKNOWLEDGMENTS}

We thank M.S. Zolotorev, B.A. Shadwick, L. Archambault, M. Dickinson and S. DiMaggio for their contributions. This research was funded by the Department of Energy, Grant No. DE-AC-03-76SF0098, DE-AC03-76SF00515, DE-AC-

03-76SF0098 and DE-FG03-98-DP-00211, National Science Foundation Contracts ECS9617089 and DMS-9722171 and Lawrence Livermore National Laboratory Contract No. WO7405-ENG-48. 


\section{REFERENCES}

1. For a review, see E. Esarey et al., "Overview of Plasma Based Accelerator Concepts," IEEE Trans. on Plas. Sci. 24, p. 252-288 (1996).

2. J. B. Rosenzweig, "Acceleration and focusing of electrons in two-dimensional nonlinear plasma wakefields," Phys. Rev. A 44, p 6854-6861 (1991). N. Barov et al., "Propagation of short electron pulses in a plasma channel," Phys. Rev. Lett. 80, p. 81-4 (1998)

3. P. Catravas et al., "Measurements of radiation near an atomic spectral line from the interaction of a $30 \mathrm{GeV}$ electron beam and a long plasma", submitted to Phys. Rev. E.

4. W.K.H. Panofsky and M. Phillips, Classical Electricity and Magnetism, AddisonWesley Publishing Company, Inc., Reading MA., 1962.

5. M.J. Hogan et al. "E-157: A 1.4-m-long plasma wake field acceleration experiment using a $30 \mathrm{GeV}$ electron beam from the Stanford Linear Accelerator Center Linac," Phys. Plasmas 7, 2241 (2000).

6. P. Muggli et al., "Photo-ionized plasma source for plasma accelerator applications," IEEE Trans. Plas. Sci 27, p. 791 (1999).

7. S. DiMaggio et al., "Development of one meter Lithium plasma source and Excimer mode reduction for plasma wakefield applications," Proc. 1999 Particle Accelerator Conference, New York, p. 3705 (1999).

8. P. Catravas et al., "Beam profile measurement at $30 \mathrm{GeV}$ using optical transition radiation," Proc. 1999 Particle Accelerator Conference, New York, p. 2111 (1999).

9. M. J. Weber, Handbook of Laser Science and Technology, Vol. III, Part I, p. 205, CRC Press (1988).

10. G.B. Rothbart, J.C. Sheppard, M.A. Piestrup, R.A. Powell, R.H. Pantell, G.A. Gearhart, "Construction of a Cerenkov light source," Rev. Sci. Instr. 50, p.411-17, (1979).

11. R. Siemann, SLAC Tech Pub. ARDB-221 (2000). C. Clayton et al., in preparation.

12. Max Zolotorev, private communication. 